\title{
Evaluación del programa "Familias fuertes: amor y límites" en familias con adolescentes de 10 a 14 años
}

\author{
M. LORETO CORREA V. ${ }^{1}$, TAMARA ZUBAREW G. ${ }^{1}$, \\ M. TERESA VALENZUELA M. ${ }^{2, a}$, FRANCISCA SALAS P. ${ }^{1}$
}

'Unidad de Adolescencia, División de Pediatría, Facultad de Medicina Pontificia Universidad Católica de Chile. 2Escuela de Enfermería Pontificia Universidad Católica de Chile. aEnfermera Matrona.

Fuente de apoyo financiero: División de Pediatría, Facultad de Medicina, Pontificia Universidad Católica de Chile.

Recibido el 29 de abril de 2011, aceptado el 19 de enero de 2012.

Correspondencia a: Loreto Correa V. Lira $85,5^{\circ}$ piso, Santiago- Chile.

Teléfonos: 3548066 Fax: 02-6384307

E-mail:mlcorrea@uc.cl

\section{Evaluation of the program "Strong families: love and limits" in families with teenagers aged 10 to 14 years}

Background: "Strong Families" is a family program aimed at preventing risk behaviors in adolescents from 10 to 14 years of age. It has been developed by the Pan American Health Organization (PAHO/WHO) and is based on the Iowa Strengthening Families Program. This program has been certified and has been proven to effectively prevent adolescent drug and alcohol abuse in several countries around the globe. Aim: To evaluate the Strong Families Program toward adjusting current parenting styles, aiming to decrease risk behaviors in Chilean adolescents. Material and Methods: A quasi-experimental study involving 120 families, selected from 6 schools within the Metropolitan Region of Santiago, Chile. A group of 129 adolescents and 124 parents were chosen by convenience to be intervened. A control group of 223 adolescents and 165 parents were not intervened. The families that underwent intervention attended to 7 educational sessions. The intervened and control groups were evaluated before intervention as well as 6 months after intervention, by means of self-administered evaluation tools. Results: The intervened parents showed significant parenting changes six months after intervention, which resulted in less yelling $(p=0.007)$, insults $(p=0.002)$ and lack of control when their children misbehaved $(p=0.008)$. Regarding the risk behaviors in the intervened and control adolescents, no changes were observed in terms of the consumption rate of tobacco, alcohol or illegal drug use, or in sexual risk behaviors. Conclusions: After six months of intervention, the Program proved to be effective in modifying parenting styles. However, no changes were observed in risk behaviors among adolescents, which could occur within a longer period of time, as reported in other studies.

(Rev Med Chile 2012; 140: 726-731).

Key words: Adolescent; Program evaluation; Risk reduction behavior.
L

os problemas de salud mental y las conductas de riesgo en adolescentes escolares determinan un deterioro considerable en la salud y desarrollo integral de éstos, afectando su calidad de vida, la de sus familias, dificultando su inserción en la sociedad y restándole la posibilidad de acceder con igualdad de oportunidades al contexto educativo. Una vez establecido el deterioro de la salud mental o la consolidación de las conductas de riesgo y sus consecuencias en los adolescentes, el cuidado y tratamiento genera un aumento de las demandas de atención en el sistema de salud, encareciendo significativamente los costos. Además, un problema que inicialmente es de salud propiamente tal, afecta directamente a la educación, perjudicando el rendimiento académico, aumentando 
las cifras de deserción y de repitencia escolar, por lo que también aumenta los recursos necesarios para llegar a un mismo logro académico ${ }^{1-3}$. De lo anteriormente expuesto surge la necesidad de realizar intervenciones costo-efectivas para reducir estos graves problemas que afectan a los adolescentes, sus familias y la sociedad entera ${ }^{4}$.

Diferentes estudios demuestran que cuando los padres o adultos referentes presentan dos cualidades básicas como son disciplina consistente y apoyo emocional, los adolescentes tienen menos problemas y conductas de riesgo ${ }^{4-8}$.

La Organización Panamericana de la Salud (OPS/OMS), en conjunto con el Centro de Comunicación en Salud, de Harvard School of Public Health, Estados Unidos de Norteamérica, ha desarrollado un programa de intervención familiar para la prevención de conductas de riesgo entre los jóvenes, basado en el programa "Iowa Strengthening Families Program" (ISFP), de la Iowa State University como una intervención primaria efectiva para prevenir el abuso de alcohol y otras sustancias psicotrópicas ${ }^{9-16}$.

El programa se llama "Familias Fuertes: Fortalecimiento de familias para prevenir conductas de riesgo en adolescentes" y cuenta con el apoyo de la OPS/OMS a través del aporte en capacitación y material educativo. Está orientado a familias a través del trabajo con padres y adolescentes de 10 a 14 años para entregarles herramientas efectivas que les permitan establecer vínculos afectivos y límites claros, que se sustentan en los conceptos de Amor y Límites ${ }^{17}$.

El programa educativo interviene a nivel parental promoviendo estilos de crianza positivos, incluyendo habilidades de comunicación, potenciación de vínculos efectivos con los hijos, la elaboración de normas de conductas y maneras adecuadas de manejar la indisciplina. En los adolescentes se promueven habilidades de resistencia a presión de pares, relaciones de pares prosociales, manejo positivo de emociones y empatía con los padres.

Este programa ha sido adaptado a la realidad social y cultural latinoamericana, en un esfuerzo conjunto de la OPS/OMS y la Universidad Estatal de Iowa, siendo validado en Latinoamérica: Perú, Guatemala, El Salvador, Nicaragua y Honduras ${ }^{18,19}$.

El propósito de este estudio es evaluar el Programa "Familias Fuertes: Amor y Límites" como herramienta metodológica para la crianza efectiva y prevención de consumo de tabaco, alcohol, drogas y actividad sexual en adolescentes entre 10 y 14 años, de distintos colegios en la Región Metropolitana en Santiago de Chile.

\section{Metodología}

Estudio cuasi-experimental con un grupo intervenido de 129 adolescentes y 124 apoderados (padres, madres o tutores), elegidos por conveniencia, a las que se les aplicó el Programa Familias Fuertes, entre los meses de agosto y noviembre de 2009, y un grupo control de 223 adolescentes y 165 apoderados, de similares características, de los mismos colegios intervenidos, a los que no se les aplicó el Taller Familias Fuertes.

El Programa Familias Fuertes incluyó: capacitación de 30 profesores monitores, realización del Taller Familias Fuertes y aplicación de instrumentos de evaluación al grupo intervenido y control en 2 momentos: preintervención y a los 6 meses de seguimiento.

Los objetivos y actividades de las sesiones del Taller para apoderados fueron dirigidas a: Aumentar su conocimiento y capacidad de resolver los conflictos con sus hijos, desarrollar su capacidad de demostrar afecto y establecer reglas claras de conducta, aumentar su capacidad para vigilar el comportamiento y desarrollar la capacidad para escuchar y comprender los problemas específicos de sus hijos. Para los adolescentes: Elegir compañías adecuadas, desarrollar mayor capacidad de resistencia a la presión de los compañeros, manejar el estrés y evitar conductas de riesgo.

Los talleres se realizaron en grupos de $20 \mathrm{fa}$ milias con al menos 4 facilitadores a cargo, en 3 colegios particulares y 3 particulares subvencionados de la Región Metropolitana de Santiago, pertenecientes a las comunas de La Pintana, La Florida, Nuñoa y Santiago Centro. El Taller constó de 7 sesiones semanales, de 3 horas cada una, con sesiones paralelas con los padres y adolescentes, que concluyen con una actividad común para la familia. La metodología utilizada fue de tipo participativa con análisis de vídeos.

El horario fue después de la jornada escolar y se realizó en el mismo colegio.

Se aplicaron instrumentos de evaluación, validados por OPS/OMS y GTZ (Corporación Técnico Alemana) para América Latina ${ }^{14,15}$, al grupo intervenido y control en 2 momentos: preintervención y a los 6 meses de seguimiento, donde 
se evaluaron los cambios en el estilo de crianza de los padres, madres o tutores y las conductas de riesgo de los adolescentes. Estos fueron de carácter anónimo y autoadministrados.

El instrumento utilizado para los padres constó de 9 preguntas con escala de respuesta tipo Likert, que describen la relación padres-hijos de acuerdo a la percepción de los padres/madres o encargados. Evaluó las manifestaciones de aprecio y cuidado hacia los hijos y la presencia de maltrato frente al compartamiento inadecuado de los hijos. El instrumento para los adolescentes constó de 122 preguntas con escala de respuesta tipo Likert, que conforman 11 sub-escalas que midieron cercanía emocional del adolescente con su familia, frecuencia de la exigencia de cumplimiento de las normas familiares, relaciones interpersonales entre padres y adolescentes, uso de drogas, tabaco y alcohol por parte de los adolescente y otras conductas de riesgo en el ámbito de la sexualidad.

Para evaluar el grado de satisfacción de los padres y adolescentes intervenidos con el Programa Familias Fuertes, al término del taller se aplicó, tanto a los adolescentes como a los padres, un cuestionario autoadministrado y anónimo, con respuesta escala tipo Likert, validado previamente por OPS Y GTZ ${ }^{14,15}$. El instrumento incluyó 7 preguntas de valoración global del programa y los contenidos, coherencia con los intereses propios, comprensión de los contenidos, grado de aprendizaje, utilidad para la vida futura y cumplimiento de expectativas.

La recolección de datos se realizó en planilla Excel y para el análisis estadístico se uso el programa SPSS 18.00 con un nivel de significancia de 5\%. Se llevó a cabo análisis descriptivo de las variables de interés. Para diferencias entre grupos casos y control se consideró el de Test de Fisher y $\chi^{2}$.

El proyecto fue aprobado por el Comité de Ética Institucional. Todas las familias, tanto intervenidas como control firmaron previamente un consentimiento informado.

\section{Resultados}

\section{Apoderados}

El grupo Padres Familias Fuertes inicial estuvo conformado por 124 apoderados que completaron la intervención y el grupo Control por 165 apoderados. En los aspectos biodemográficos, no hubo diferencias entre el grupo intervenido y el grupo control. El grupo intervenido presenta una edad promedio de 43,4 y el grupo control de 41,7 años. El 62\% del grupo padres Familias Fuertes fue de sexo femenino, mientras que en el grupo Padres Control fue de $58 \%$.

Ambos grupos, intervenido y control, no mostraron diferencias estadísticamente significativas en los estilos de crianza de sus hijos adolescentes antes de la intervención.

Respecto de las variables que describen la relación de crianza de acuerdo a la percepción de los padres/madres y/o tutores postintervención se observó:

En los ítems que evaluaron manifestaciones de aprecio y cuidado hacia los hijos se evidenció una tendencia favorable en el grupo padres intervenidos versus el grupo control, pero no estadísticamente significativa.

En los ítems que evaluaron maltrato ante el comportamiento de los hijos se observó cambios estadísticamente significativos en los padres intervenidos versus el grupo control: disminuyó el uso de los gritos como respuesta al mal comportamiento de los hijos, disminuyó el uso de insultos frente a un comportamiento inadecuado de los hijos y disminuyó la falta de control de los padres frente al mal comportamiento de los hijos. (Tabla 1).

\section{Adolescentes}

El grupo intervenido estuvo conformado por 129 adolescentes y el grupo control por 223 adolescentes. En los aspectos biodemográficos, no hubo diferencias entre ambos grupos. El grupo intervenido presentaba una edad promedio de 11,5 años y el grupo control 11,6 años. Cincuenta y nueve porciento del grupo intervenido era femenino, mientras que en el grupo control fue de $57 \%$. En ambos grupos el $67 \%$ vivía con sus dos padres, $90 \%$ de los adolescentes grupo intervenido tenía hermanos, similar al 88\% de los adolescentes control .

En la evaluación inicial no hubo diferencias significativas respecto de aspectos relacionales, actitudinales ni en las conductas de riesgo entre ambos grupos.

Con respecto a las conductas de riesgo evaluadas a los 6 meses post-intervención, no se evidenciaron cambios estadísticamente significativos en consumo de tabaco, alcohol, drogas ilícitas ni conductas sexuales de riesgo. 
Familias fuertes: amor y límites - M. L. Correa et al

Tabla 1. Estilos de Crianza en Padres Familias Fuertes y Control

\begin{tabular}{|c|c|c|c|c|c|c|}
\hline & Co & rol & $\mathbf{P}$ & & rte & $\mathbf{P}$ \\
\hline & $\mathbf{0}$ & 6 & & $\mathbf{0}$ & 6 & \\
\hline & $\%$ & $\%$ & & $\%$ & $\%$ & \\
\hline ¿Le gritó a su hijo/a porque estaba enojada/o con él/ella? & & & NS & & & 0,007 \\
\hline Nunca & 17,0 & 19,8 & & 12,9 & 24,2 & \\
\hline Pocas & 64,8 & 62,9 & & 54,8 & 60,4 & \\
\hline Muchas & 17,6 & 17,2 & & 29,0 & 13,2 & \\
\hline Siempre & 0,6 & 0,0 & & 3,2 & 2,2 & \\
\hline Sin Resp & 0,0 & 0,0 & & 0,0 & 0,0 & \\
\hline ¿Le gritó o insultó cuando no estaban de acuerdo con su hijo/a? & & & NS & & & 0,002 \\
\hline Nunca & 58,8 & 69,0 & & 43,5 & 65,9 & \\
\hline Pocas & 33,3 & 27,6 & & 42,7 & 29,7 & \\
\hline Muchas & 5,5 & 1,7 & & 13,7 & 3,3 & \\
\hline Siempre & 1,2 & 0,0 & & 0,0 & 1,1 & \\
\hline Sin Resp & 1,2 & 1,7 & & 0,0 & 0,0 & \\
\hline ¿Cuándo su hijo/a hizo algo mal, usted perdió los nervios ...? & & & NS & & & 0,008 \\
\hline Nunca & 37,0 & 45,7 & & 23,4 & 39,6 & \\
\hline Pocas & 50,9 & 46,6 & & 53,2 & 50,5 & \\
\hline Muchas & 10,3 & 7,8 & & 21,8 & 8,8 & \\
\hline Siempre & 1,2 & 0,0 & & 0,8 & 1,1 & \\
\hline Sin Resp & 0,6 & 0,0 & & 0,8 & 0,0 & \\
\hline
\end{tabular}

NS ( No Significativa).

Se observó una adherencia de 100\% de participación de las familias hasta el final de las 7 sesiones del taller. Sin embargo, en las encuestas de seguimiento realizada a los 6 meses post taller se obtuvo $73 \%$ de respuestas en los apoderados intervenidos y $70 \%$ en los controles. En los adolescentes el porcentaje de respuestas alcanzó el 82\% $y$ en el control $78 \%$.

\section{Grado de satisfacción con el taller}

Se encuestó al $86 \%$ de los padres y $84 \%$ de los adolescentes al término del taller. Noventa y ocho porciento de los apoderados y adolescentes refirieron alta satisfacción con la calidad del taller.

\section{Discusión y conclusiones}

Múltiples estudios, tanto nacionales como internacionales, demuestran el efecto protector de un adecuado vínculo familiar sobre la disminución de las conductas de riesgo en los adolescentes ${ }^{2,5-8}$. En nuestro país se ha descrito que todas las conductas de riesgo son más frecuentes en adolescentes que perciben a sus familias como disfuncionales y enfatiza la necesidad de implementar acciones orientadas al refuerzo de la comunicación y fortalecimiento de lazos familiares con adolescentes ${ }^{20}$.

El Programa Familias Fuertes se comenzó a aplicar en algunos colegios en Chile desde el 2005, sin embargo, por primera vez este estudio evalúa el impacto de la intervención. A seis meses de seguimiento demuestra resultados significativos en el fortalecimiento de estilos de crianza efectivos y comunicación padre/hijo, donde se observa disminución del uso de violencia verbal y mejor control de padres ante el mal comportamiento de sus hijos.

No se observaron diferencias en las conductas de riesgo de los adolescentes intervenidos, lo que puede atribuirse a que la intervención se realizó a edades tempranas, promedio 11 años, por lo que aún la mayor parte de estos adolescentes no ha comenzado a experimentar conductas de riesgo ${ }^{3}$. Se espera que las diferencias entre ambos grupos aparezcan más adelante en el seguimiento, tal como se describe en los estudios de evaluación del programa ISFP $4,9,11,15$.

En general, la literatura describe elevadas pérdidas de familias durante el transcurso de los talleres para padres ${ }^{14,21,22}$, sin embargo, este estudio 
presentó alta adherencia, lo que puede ser explicado por las atractivas metodologías y la motivación de los facilitadores, lo que se vio reflejado en el alto grado de satisfacción de adolescentes y sus apoderados.

No hay publicaciones nacionales que muestren evidencias del efecto de talleres de fortalecimiento familiar en la disminución de conductas de riesgo de adolescentes. Sin embargo, el programa ISFP en estudios internacionales ha demostrado un efecto progresivo en la disminución de variadas conductas de riesgo en los adolescentes intervenidos versus grupo control, hasta 6 años de seguimiento ${ }^{9,11}$.

Este estudio presentó algunas limitaciones tales como: los controles son alumnos de los mismos colegios intervenidos, por lo que puede existir cierto grado de contaminación de los efectos del taller en los alumnos y apoderados no intervenidos. Además es posible que existan otros programas de intervención simultáneos en los colegios que apunten a los mismos objetivos del Taller Familias Fuertes, lo que pudiera verse reflejado en que el grupo control muestre mejoría en algunas variables de crianza parental. Por último, existió una pérdida de respuestas en el seguimiento a 6 meses post taller tanto en el grupo control como intervenido. Esto requiere buscar estrategias que permitan aminorar esta deficiencia.

En materia de salud, Chile considera dentro de las Políticas Públicas de protección social, disponer de un sistema de salud capaz de anticiparse a los problemas sanitarios ambientales a través de estrategias de promoción y prevención ${ }^{23}$. Dado lo anterior es que cobra gran relevancia este tipo de intervención intersectorial e interdisciplinaria, ya que contribuye desde las distintas miradas de salud y educación a prevenir algunos de los problemas que aquejan a los adolescentes de nuestro país como son el abuso de alcohol y otras sustancias psicotrópicas y ayuda a promover un desarrollo social saludable en este grupo etario y sus familias.

Una de las fortalezas del estudio es que permitió capacitar a los profesores de los distintos colegios participantes, trabajar colaborativamente con ellos y dejar la capacidad instalada en los colegios para seguir replicando el Taller en el tiempo.

\section{Referencias}

1. United Nations Children's Fund. The State of the World's Children 2011. Adolescence: and Age of Oppor- tunity [Internet]. New York: UNICEF; 2011 [citado 10 de agosto de 2011]. Disponible en: http://www.unicef. org/publications/index_57468.html

2. Breinbauer C, Maddaleno M. Estilos de Vida de los Adolescentes de América Latina y el Caribe: Desafíos y alcances. En: Organización Panamericana de la Salud. Jóvenes: Opciones y Cambios: Promoción de Conductas Saludables en los Adolescentes. Washington DC: OPS, 2008; 5-11.

3. Ministerio de Salud (Chile). Encuesta Mundial de Salud Escolar 2004 y 2005 [Internet]. Santiago, Chile: MINSAL; 2007 [citado 10 de abril de 2011]. Disponible en: http://epi.minsal.cl/epi/html/invest/EMSE/INFORME\%20EMSE\%202004-2005\%20final.pdf

4. Spoth R, Guyll M, Day S. Universal Family-Focused Interventions in Alcohol-Use Disorder Prevention: Cost-Effectiveness and Cost-Benefit Anlyses of Two Interventions. J Stud Alcohol 2002; 63: 219-28.

5. Simpson A. Ser padres de adolescentes: Una síntesis de la investigación y una base para la acción [Internet]. Washington, DC: OPS; 2004 [citado 10 de abril de 2011]. Disponible en http://www.paho.org/Spanish/AD/FCH/ CA/serpadresadol.pdf

6. Barber B, Schluterman J. Connectedness in the lives of children and adolescents: a call for greater conceptual clarity. J Adolesc Health 2008; 43 (3): 209-16.

7. Florenzano R, Valdés M, Cáceres E, Casassus M, Sandoval A, Santander S, et al. Percepción de la relación parental entre adolescentes mayores y menores de 15 años. Rev Chil Pediatr 2009; 80 (6): 520-7.

8. Florenzano R. El Adolescente y sus conductas de Riesgo. $3^{a}$ ed. Santiago, Chile: Pontificia Universidad Católica de Chile; 2005.

9. Spoth R, Trudeau L, Chungyeol S. Long-term effects of universal Preventive Interventions on Prescription Drug Misuse. Addiction 2008; 103: 1160-8.

10. Spoth R, Chungyeol S, Guyll M. Universality of Effects: An Examination of the Comparability of Long-Term Family Intervention Effects on Substance Use Across Risk-Relate Subgroups. Prev Sci 2006; 7: 209-24.

11. Spoth R, Guyll M, Chungyeol S. Universal Intervention as a Protective Shield Against Exposure to Substance Use: Long Term Outcomes and Public Health Significance. Am J Public Health 2009; 99 (11): 2026-33.

12. Guyll M, Spoth R, Chao W, Wickrama K, Russell D. Family-Focused Preventive Interventions: Evaluating Parental Risk Moderation of Substance Use Trajectories. J Fam Psychol 2004; 18 (2): 293-301.

13. Spoth R, Redmond C, Shin C, Azevedo K. Brief Family Intervention Effects on Adolescent Substance Initiation: School-level Curvilinear Growth Curve Analyses Six 
Years Following Baseline. J Consult Clin Psychol 2004; 72 (3): 535-42.

14. Redmond C, Spoth R, Shin C, Hill G. Engaging Rural Parents in Family-Focused Programs to Prevent Youth Substance Abuse. J Prim Prev 2004; 24 (3): 223-42.

15. Spoth R, Redmond C. Project Family Prevention Trials Based in Community-University Partnerships: Toward Scale Up Preventive Interventions. Prev Sci 2002; 3 (3): 203-21.

16. Spoth R, Redmond C, Shin C. Randomized Trial of Brief Family Interventions for General Populations: Adolescent Substance use Outcomes 4 years Following Baseline. J Consult Clin Psychol 2001; 69 (4): 627-42.

17. Organización Panamericana de la Salud. Familias Fuertes: Programa Familiar para Prevenir Conductas de Riesgo en Jóvenes. Guía para el Facilitador. Washington DC. OPS. USA. 2006.

18. Azziz-Baumgartner C, Wilson LL. Applying a Model of Program Adaptation to the Familias Fuertes Parent/ Adolescent Educational Intervention for Latino Immigrant Families in the Rural South. South Online J Nurs Res [Internet]. 2009 [citado 10 de enero de 2011]; 9 (3):
[14 p.] Disponible en: http://snrs.org/publications/ SOJNR_articles2/Vol09Num03Art07.pdf

19. Kumpfer K, Pinyuchon M, Teixeira A, Whiteside HO. Cultural Adaption Process for International Dissemination of the Strengthening Families Program. Eval Health Prof 2008; 31(2): 226-39.

20. Santander S, Zubarew T, Santelices L. Influencia de la familia como factor protector de conductas de riesgo en escolares chilenos. Rev Med Chile 2008; 136: 317-24.

21. Errasti P, Al- Halabi D, Secades V, Fernández-Hermida, Carballo J, García-Rodríguez O. Prevención Familiar del Consumo de Drogas: el Programa "Familias que Funcionan". Psicothema 2009; 21 (1): 45-6.

22. Kane GA, Wood VA, Barlow J. Parenting programmes: a systematic review and synthesis of qualitative research. Child Care Health Dev 2007; 33 (6): 784-93.

23. Williams G. Descripción de Algunas Políticas Públicas en Chile [Internet]. Santiago, Chile: Biblioteca del Congreso Nacional ; 2006 [ citado 10 de abril de 2011] Disponible en: http://www.bcn.cl/carpeta_temas/temas_portada.2006-0725.7747914711/documentos_pdf.2006-0725.1270524036 . 